\title{
A loss of $P d x k$ model of Parkinson disease in Drosophila can be suppressed by Buffy
}

P. Githure M'Angale and Brian E. Staveley*

\begin{abstract}
Background: The identification of a DNA variant in pyridoxal kinase ( $P d x k$ ) associated with increased risk to Parkinson disease (PD) gene led us to study the inhibition of this gene in the Dopa decarboxylase (Ddc)-expressing neurons of the well-studied model organism Drosophila melanogaster. The multitude of biological functions attributable to the vitamers catalysed by this kinase reveal an overabundance of possible links to PD, that include dopamine synthesis, antioxidant activity and mitochondrial function. Drosophila possesses a single homologue of $P d x k$ and we used RNA interference to inhibit the activity of this kinase in the Ddc-Gal4-expressing neurons. We further investigated any association between this enhanced disease risk gene with the established PD model induced by expression of $a$-synuclein in the same neurons. We relied on the pro-survival functions of Buffy, an anti-apoptotic BCl-2 homologue, to rescue the Pdxk-induced phenotypes.

Results: To drive the expression of Pdxk RNA interference in DA neurons of Drosophila, we used Ddc-Gal4 which drives expression in both dopaminergic and serotonergic neurons, to result in decreased longevity and compromised climbing ability, phenotypes that are strongly associated with Drosophila models of PD. The inhibition of Pdxk in the a-synuclein-induced Drosophila model of PD did not alter longevity and climbing ability of these flies. It has been previously shown that deficiency in vitamers lead to mitochondrial dysfunction and neuronal decay, therefore, coexpression of $P d x k$-RNAi with the sole pro-survival Bcl-2 homologue Buffy in the Ddc-Gal4-expressing neurons, resulted in increased survival and a restored climbing ability. In a similar manner, when we inhibited $P d x k$ in the developing eye using GMR-Gal4, we found that there was a decrease in the number of ommatidia and the disruption of the ommatidial array was more pronounced. When $P d x k$ was inhibited with the a-synuclein-induced developmental eye defects, the eye phenotypes were unaltered. Interestingly co-expression with Buffy restored ommatidia number and decreased the severity of disruption of the ommatidial array.
\end{abstract}

Conclusions: Though Pdxk is not a confirmed Parkinson disease gene, the inhibition of this kinase recapitulated the PD-like symptoms of decreased lifespan and loss of locomotor function, possibly producing a new model of PD.

Keywords: Dopaminergic neurons, Drosophila, Parkinson disease, Pyridoxal kinase, Pyridoxal 5' phosphate, Buffy

\section{Background}

Parkinson disease (PD), the second most common human neurodegenerative disorder, after Alzheimer disease, afflicts about $1 \%$ of the population over the age of 50 years of age [1]. The clinical disorders associated with PD are a variable combination of bradykinesia,

${ }^{*}$ Correspondence: bestave@mun.ca

Department of Biology, Memorial University of Newfoundland, St. John's, NL A1B 3X9, Canada postural instability, tremor, and rigidity, with a typical positive response to Levodopa [2]. Non-motor symptoms that include autonomic dysfunction, cognitive decline and psychiatric problems can also present [3]. These end-points are mainly attributed to the loss of dopaminergic (DA) neurons of the substantia nigra with the degeneration of the nigrostriatal dopaminergic system. However, the neuropathology of PD is known to be more widespread, with many non-dopaminergic nuclei 
affected, including the locus coeruleus, the brain stem, raphe nucleus, dorsal motor nucleus of the vagus, basal nucleus of the Meynert, amygdala, and hippocampus [4]. PD is characterized by the presence of neuronal inclusions composed of abnormal $\alpha$-synuclein and generally referred to as Lewy-related pathology $[2,5]$. This atypical protein accumulation is believed to lead to cellular toxicity and, eventually, the PD pathogenesis. A majority of PD cases are idiopathic but the emergence of familial cases led to the identification and study of genes that are highly associated with PD $[6,7]$. The understanding and exploitation of the genetic basis of PD has revealed over 20 genes that are implicated in PD pathogenesis [8], and highlighted the complexity of this neurodegenerative disease.

The link between vitamin $\mathrm{B}_{6}$ and PD incidence has been explored for years, with some studies associating dietary vitamin $B_{6}$ with reduced effectiveness of Levodopa [9]. Other studies have shown the advantages of a higher dietary vitamin $\mathrm{B}_{6}$ and the reduced risk of PD [10] or reported low dietary intake of vitamin $B_{6}$ with increased risk to PD [11], either via its antioxidant abilities or through dopamine biosynthesis. Vitamin $\mathrm{B}_{6}$ is comprised of three pyridine derivatives or vitamers - which are chemical compounds that have a similar molecular structure and possess similar vitamin activity-known as pyridoxine (PN), pyridoxamine (PM), pyridoxal (PL) and their phosphorylated products pyridoxine- $5^{\prime}$-phosphate (PNP), pyridoxamine- $5^{\prime}$-phosphate (PMP) and pyridoxal$5^{\prime}$-phosphate (PLP) $[12,13]$. PLP is the most metabolically active form and responsible for more than 100 enzymatic reactions [12], predominantly in amino acid metabolism, and is implicated in nervous system function (neurotransmitter synthesis), red blood cell formation (heme biosynthesis), vitamin formation, one-carbon metabolism (nucleic acid synthesis) and as a potent antioxidant [14]. In neuronal function, PLP plays a key role in the metabolism of neurotransmitters, including dopamine, serotonin, glycine, GABA, glutamate, D-serine and histamine [12]. The deficiency of vitamin $B_{6}$ has been implicated in increased risk of cancer, neural decay and accelerated ageing. Mitochondrial oxidative decay is a major contributor to ageing $[15,16]$. Mitochondrial function is more dependent on PLP than any other organelle as PLP function as a coenzyme for transaminases that are involved in the catabolism of all amino acids by the urea cycle of the mitochondria [16]. PLP is involved in diverse biochemically important roles in the mitochondria including maintaining energy pathways, homocysteine and glutathione (an antioxidant) biosynthesis. The heme biosynthesis occurs predominantly in the mitochondria and depends on PLP as a coenzyme. The inadequate synthesis of heme can cause mitochondrial decay and oxidative DNA damage [15], whereas its inhibition can cause oxidant leakage, that increases cellular endogenous ROS formation. Vitamin $\mathrm{B}_{6}$ has a direct antioxidant activity by preventing superoxide radical formation, glycated haemoglobin formation and erythrocyte lipid peroxidation [17]. The inter-conversion of the pyridines to the biologically active phosphate derivative PLP require the action of pyridoxal kinase, thus, the activation of vitamin $\mathrm{B}_{6}$ to its active form, PLP, requires pyridoxal kinase.

Pyridoxal kinase (Pdxk) belongs to the phosphotransferase family of proteins that are involved in the phosphorylation of vitamin $\mathrm{B}_{6}$ to pyridoxal-5-phosphate an important co-factor in intermediary metabolism [18, 19]. They contain a ribokinase/pyridoxal domain and are highly conserved, being found in yeast, plants and animals. The association of the gene coding for $P d x k$ with Parkinson disease was through whole-genome expression profiling of human DA neurons, combined with association analysis in differentially regulated genes [20]. A DNA variant, single nucleotide polymorphism, in the $P d x k$ gene has been associated with an increased risk to PD [20], though other studies ruled out the association of the variant rs2010795 with PD in a cohort of patients [21]. The study did not rule out the existence of the Pdxk variants that may increase the risk for PD. The development of model systems to study potential therapies is important, and as such Drosophila melanogaster is a good model organism to study the pathophysiology of movement disorders [22]. The first Drosophila model of PD relied on the expression of human $\alpha$-synucleinwhich has no known homologue in Drosophila-to induce the PD-like symptoms [23]. Its success anchors on the ability to recapitulate features of human PD such as (1) age-dependent loss in locomotor function (2) LBlike inclusions and (3) age-dependent loss of DA neurons; and is widely used in the study of $\alpha$-synuclein-induced neurodegeneration [22-27]. The utilization of the UAS/ GAL4 spatio-temporal expression system [28], and the availability of a plethora of promoters or enhancers of which TH-Gal4, elav-Gal4 and Ddc-Gal4 are employed in modelling PD in flies, makes Drosophila a powerful model organism [22-27]. We have previously shown the pro-survival advantages of Buffy, the sole anti-apoptotic $B c l-2$ homologue in Drosophila by its rescue of the $\alpha$-synuclein-induced phenotypes [29], in the HtrA2 model of PD [30], we extended this study to investigate whether Buffy would rescue the loss of $P d x k$-induced phenotypes in Drosophila.

\section{Results}

$P d x k$ is evolutionarily conserved across diverse species

The bioinformatic analysis of protein sequences encoded by the human, mouse, mosquito and fruit fly $P d x k$ gene 
revealed a highly conserved ribokinase/pyridoxal kinase domain (Fig. 1) as determined by NCBI Conserved Domain Database (CDD) [31] and Eukaryotic linear motif (ELM) resource [32]. Comparison of the human and the Drosophila homologues by NCBI's BLAST revealed a $46 \%$ identity and $64 \%$ similarity along the protein sequences. The alignment of protein sequences using Clustal Omega multiple sequence alignment [33] demonstrates the high level of conservation of the kinase domain. The predicted Drosophila protein appears to be localised in the cytoplasm as predicted by MultiLoc2 [34], and to possess active sites and motifs that include a weak nuclear export signal (NES) as determined by NetNES [35], a nuclear localization signal (NLS) that was detected by cNLS Mapper [36], and a transmembrane (TM) domain as predicted by TMpred [37]. Motifs identified by ELM that may contribute to the function of the Drosophila homologue are ubiquitination site, protein phosphatase 1 interacting motif, IAP binding motif, and an Src Homology 2 (SH2) domain binding motif.

\section{Loss of $P d x k$ decreases lifespan and locomotor ability}

The inhibition of $P d x k$ in the Ddc-Gal4-expressing neurons by RNA interference results in flies with a decreased lifespan and impaired locomotor function. The median lifespan for $P d x k$-RNA $i$ flies was determined to be 50 days compared to 70 days for the control flies that express the lacZ transgene (Fig. 2a). When $P d x k$ was suppressed

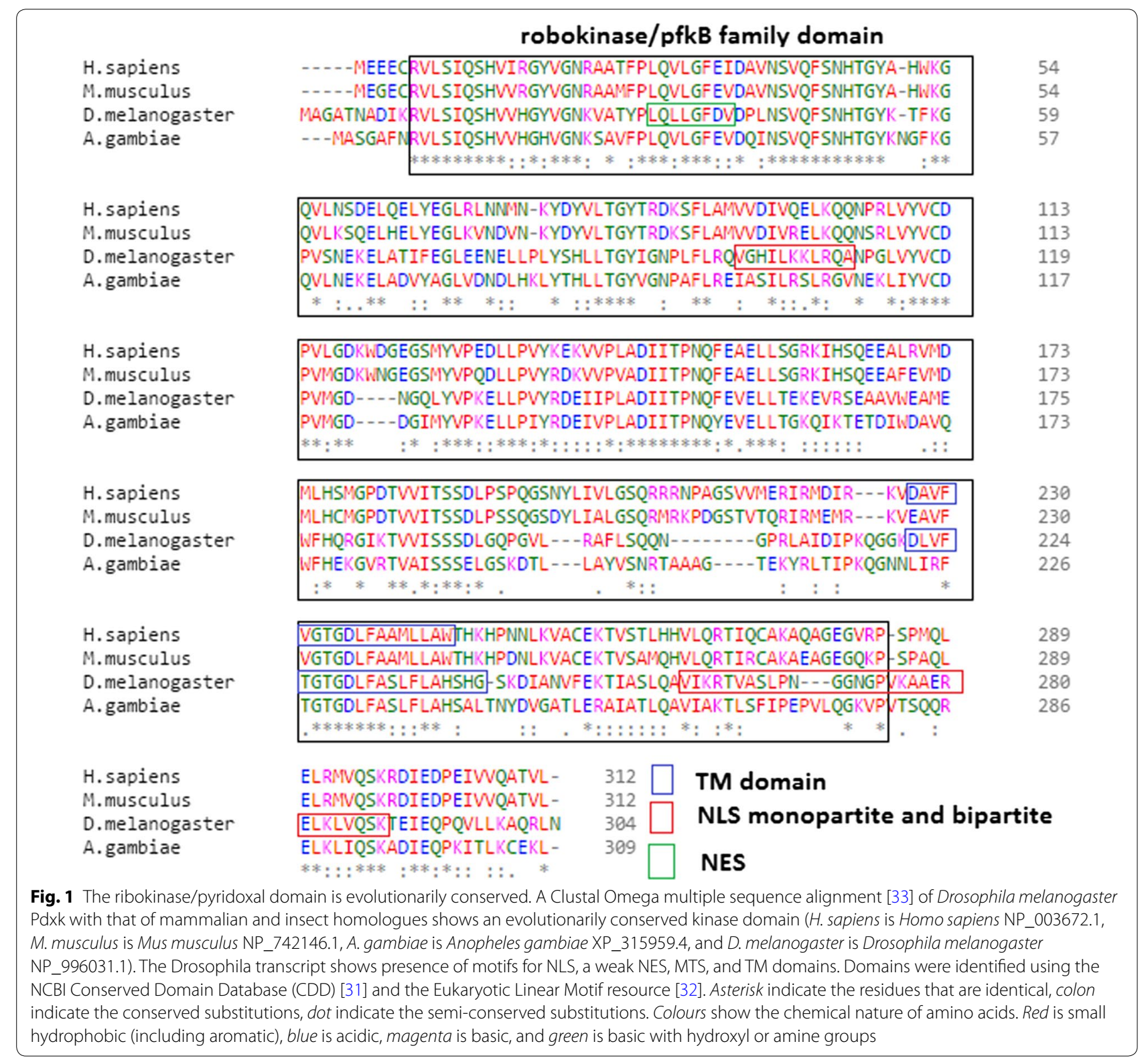


in the Ddc-Gal4-expressing neurons the flies showed impaired locomotor ability as determined by the nonlinear fitting of the climbing curves (Fig. 2b). The 95\% confidence intervals $(\mathrm{CI})$ were $0.038-0.049$ compared to 0.051-0.076 for the controls. Taken together, these results indicate an important role for this kinase in the $D d c$ Gal4-expressing neurons of Drosophila as interference with its activity phenocopies some of the well-established phenotypes observed in other Drosophila models of PD.

\section{Inhibiting $P d x k$ does not suppress $a$-synuclein-induced phenotypes}

The co-expression of $P d x k-R N A i$ with the expression of $\alpha$-synuclein does not alter the diminished longevity nor does it change the observed loss of climbing ability
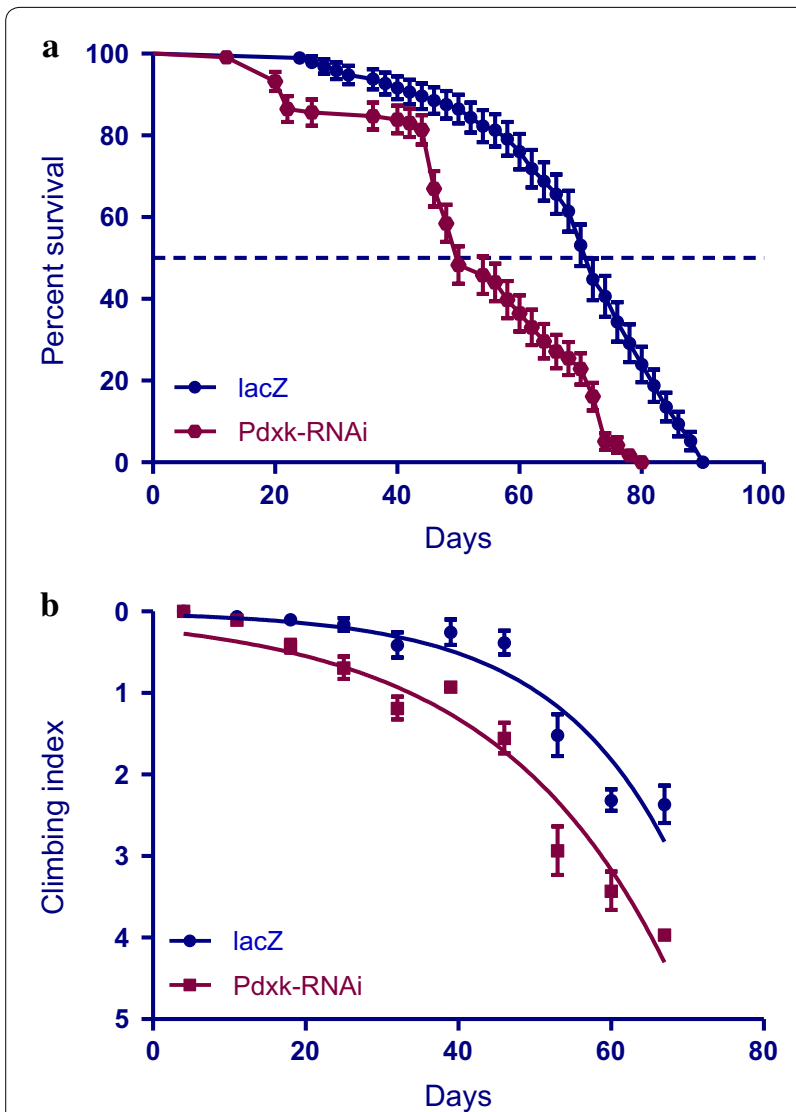

Fig. 2 Inhibiting $P d x k$ activity in Ddc-Gal4-expressing neurons decreases lifespan and impairs locomotor function. a The directed expression of $P d x k-R N A i$ in the Ddc-Gal4-expressing neurons using the $D d c-G a l 4$ transgene results in a decrease in median lifespan when compared to control flies expressing UAS-lacZ. The genotypes are UAS-lacZ/Ddc-Gal4 and Pdxk-RNAi/Ddc-Gal4. Longevity is shown as percent survival $[P<0.05$, determined by the log-rank (Mantel-Cox) test and $n \geq 200]$. b The inhibition of $P d x k$ in the Ddc-Gal4-expressing neurons resulted in a significant decrease in climbing ability as determined by nonlinear fitting of the climbing curves and comparing 95\% CI. The genotypes are UAS-lacZ/Ddc-Gal4 and Pdxk-RNAi/DdcGal4. Error bars indicate SEM and $n=50$ over time. The median lifespan was 58 days for $P d x k$ flies compared to 60 days for control flies (Fig. 3a). A comparison of the climbing curves at $95 \% \mathrm{CI}$ for Pdxk-RNAi flies was $0.033-0.045$ compared to $0.036-0.048$ for the controls. This comparison showed that they were not significantly different from each other (Fig. 3b). This implies that the inhibition of $P d x k$ in the Ddc-Gal4expressing neurons does not influence the neurotoxic effects of $\alpha$-synuclein.

\section{The pro-survival Buffy suppresses the loss of $P d x k$ phenotypes}

The co-expression of the pro-survival $\mathrm{Bcl}$-2 homologue Buffy with Pdxk-RNAi in Ddc-Gal4-expressing neurons results in a slightly increased lifespan and an improved climbing ability. The median survival of $P d x k-R N A i$ flies with co-expression of Buffy was 68 days when compared to 74 days for the controls that expressed the lac $Z$ transgene and Buffy, as determined by Log-rank at a $\mathrm{P}<0.0001$ (Fig. 4a). The climbing ability was significantly improved as determined by a nonlinear fitting of the climbing curves and compared at 95\% CI (Fig. 4b). These results suggest a pro-survival role for Buffy as exhibited by significant increases in both survival and locomotor function when $P d x k$ is inhibited in the Ddc-Gal4expressing neurons.

Inhibiting $P d x k$ in the developing eye results in disruption of the ommatidial array that can be suppressed by Buffy The inhibition of $P d x k$ in the developing eye directed by the GMR-Gal4 transgene results in eyes with a significantly lower mean number of ommatidia, at 686.4 for $P d x k-R N A i$ flies compared to 706.9 for the $l a c Z$ control flies (Fig. 5a, b) as determined by an unpaired $\mathrm{T}$ test with a $\mathrm{P}$ value of 0.0007 . More disruption of the ommatidial array was observed in Pdxk-RNAi flies, at $37.3 \%$ when compared to the lac $Z$ control flies at $25.5 \%$ (Fig. $5 \mathrm{a}, \mathrm{b}$ ) as determined by an unpaired $\mathrm{T}$ test with a $\mathrm{P}$ value of 0.0001 . The inhibition of $P d x k$ along with $\alpha$-synuclein expression did not result in marked differences in either the number of ommatidia or the disruption of the eye between the flies that express $P d x k-R N A i$ along with $\alpha$-synuclein, when compared to control flies that expressed lacZ along with $\alpha$-synuclein (Fig. 5a, c). The mean number of ommatidia for Pdxk-RNAi/ $\alpha$-synuclein flies was 643.8 compared to 675.6 for the lacZ/ $\alpha$-synuclein flies, a not significant difference as determined by an unpaired $\mathrm{T}$ test $(P=0.0616)$. Additionally, the disruption of the ommatidial array was not significantly different, at 45.8 and $49.8 \%$ respectively as determined by an unpaired $\mathrm{T}$ test $(\mathrm{P}=0.3117)$. However, a one-way analysis of variance between $P d x k-R N A i / \alpha$-synuclein and $P d x k-R N A i$ 

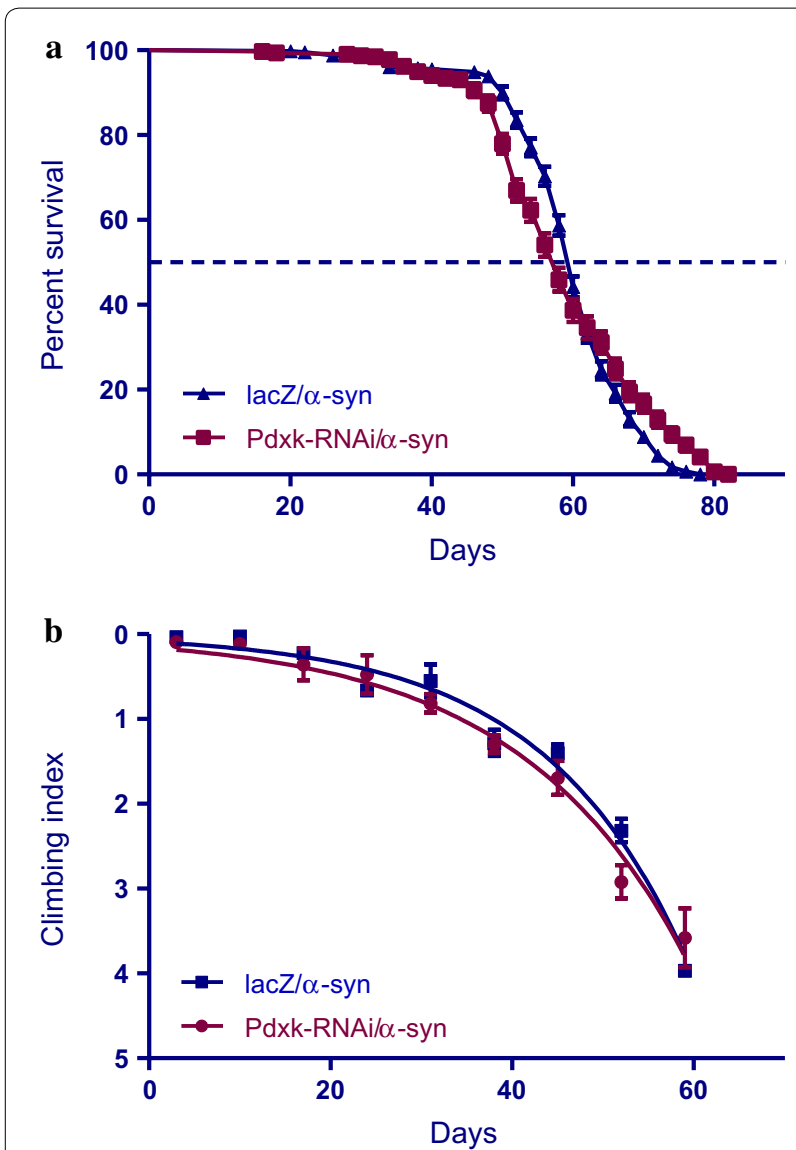

Fig. 3 Inhibition of $P d x k$ in the $a$-synuclein-induced PD model does not alter phenotypes. a The inhibition of Pdxk with $\alpha$-synuclein in the Ddc-Gal4-expressing neurons showed no significant change in lifespan when compared to the control. Genotypes are UAS- $a$ synuclein; Ddc-Gal4/UAS-lacZ and UAS-a-synuclein; Ddc-Gal4/PdxkRNAi. Longevity is shown as percent survival $[P<0.05$, determined by log-rank (Mantel-Cox) test with $n \leq 200]$. b The co-expression of $P d x k-R N A i$ in the a-synuclein model of PD did not result in any significant age-dependent loss in climbing ability compared to the control. The genotypes are UAS- $a$-synuclein; Ddc-Gal4/UAS-lacZ and UAS-a-synuclein; Ddc-Gal4/Pdxk-RNAi. Analysis was done by nonlinear fitting of the climbing curves and significance was determined by comparing the $95 \%$ confidence intervals. Error bars indicate SEM and $n=50$

flies revealed that the expression of $\alpha$-synuclein along with $P d x k-R N A i$ had a significantly severe phenotype in the eye compared to the $P d x k-R N A i$ alone flies (Fig. 5a, c), marked by decreased mean ommatidia number and higher disruption of the ommatidial array. It appears that the inhibition of $P d x k$ in the developing eye along with $\alpha$-synuclein expression enhances the $P d x k$-RNAi-induced eye phenotype. When we coexpressed $P d x k-R N A i$ with Buffy, the number of ommatidia for $P d x k-R N A i / B u f f y$ flies was 704.3 compared to 711.9 for the flies that expressed lacZ along with Buffy
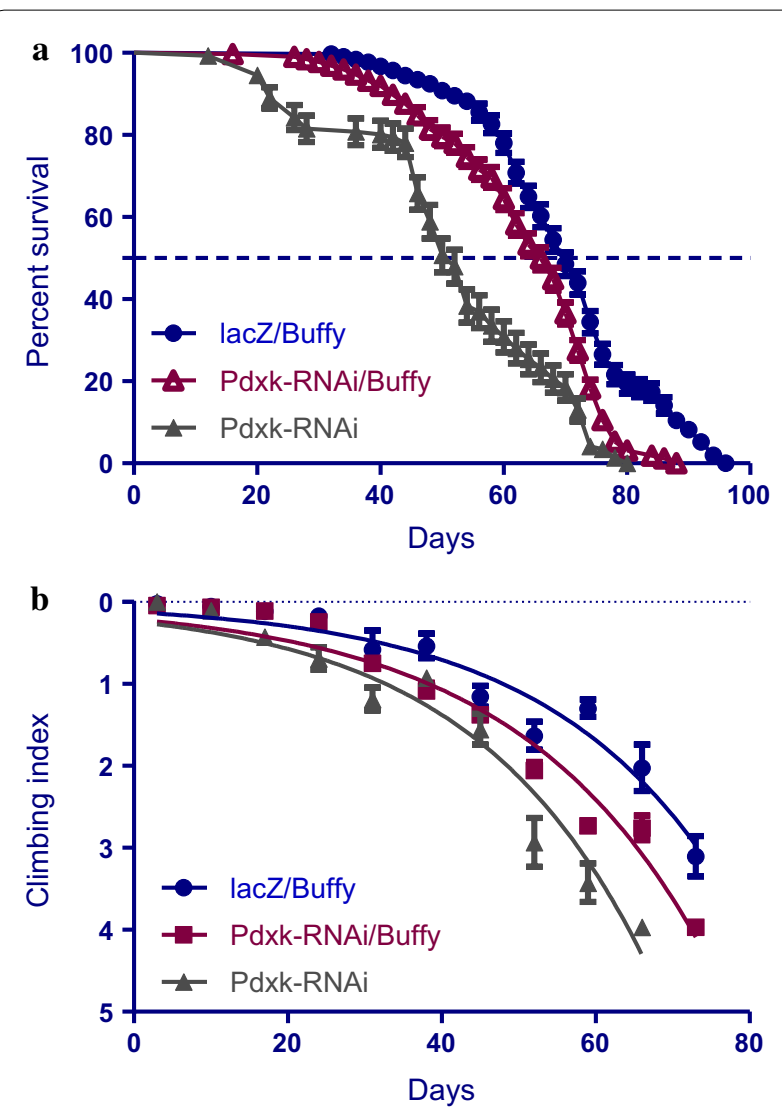

Fig. 4 The co-expression of $P d x k-R N A i$ with Buffy suppresses the $P d x k$-induced phenotypes. a The co-expression of Buffy with $P d x k-R N A i$ result in the suppression of the observed phenotype of decreased survival. Genotypes are Ddc-Gal4 UAS-Buffy/UAS-lacZ and Ddc-Gal4 UAS-Buffy/Pdxk-RNAi. Longevity is shown as percent survival $[P<0.05$, determined by log-rank (Mantel-Cox) test with $n \leq 200]$. b The co-expression of Pdxk-RNAi with Buffy in the Ddc-Gal4-expressing neurons results in the suppression of the age-dependent loss in climbing ability. The genotypes are Ddc-Gal4 UAS-Buffy/UAS-lacZ and Ddc-Gal4 UAS-Buffy/Pdxk-RNAi. Analysis was done by nonlinear fitting of the climbing curves and significance was determined by comparing the $95 \%$ confidence interval. Error bars indicate SEM and $n=50$

overexpression, this restored the $P d x k-R N A i$-induced eye phenotypes to control levels. Similarly, the disruption of the ommatidial array was down to $8.4 \%$ for $P d x k$ RNAi/Buffy flies compared to $6.4 \%$ for lacZ/Buffy flies. These comparisons were not significant as indicated by an unpaired $\mathrm{T}$ test with $\mathrm{P}$ values of 0.469 and 0.4115 respectively (Fig. 5a, d). However, a one-way analysis of variance between $P d x k-R N A i / B u f f y$ and $P d x k-R N A i$ alone flies (Fig. 5a, b) showed a remarkable recovery of the $P d x k$-RNAi-induced phenotypes. Taken together, these results suggest that Buffy suppresses the developmental eye defects resulting from the inhibition of $P d x k$ in the developing eye. 


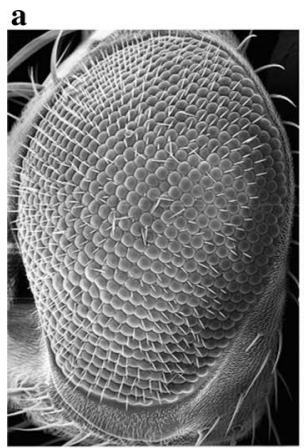

lacZ

b

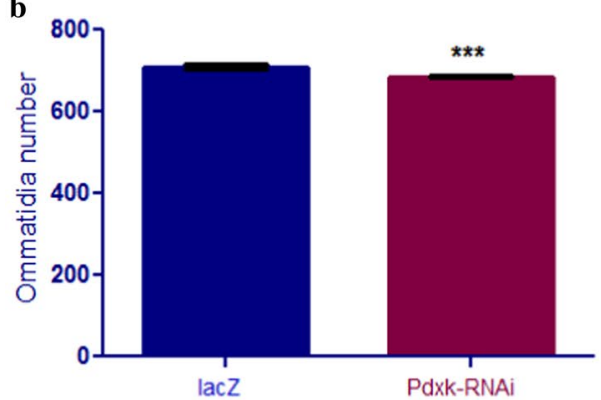

c
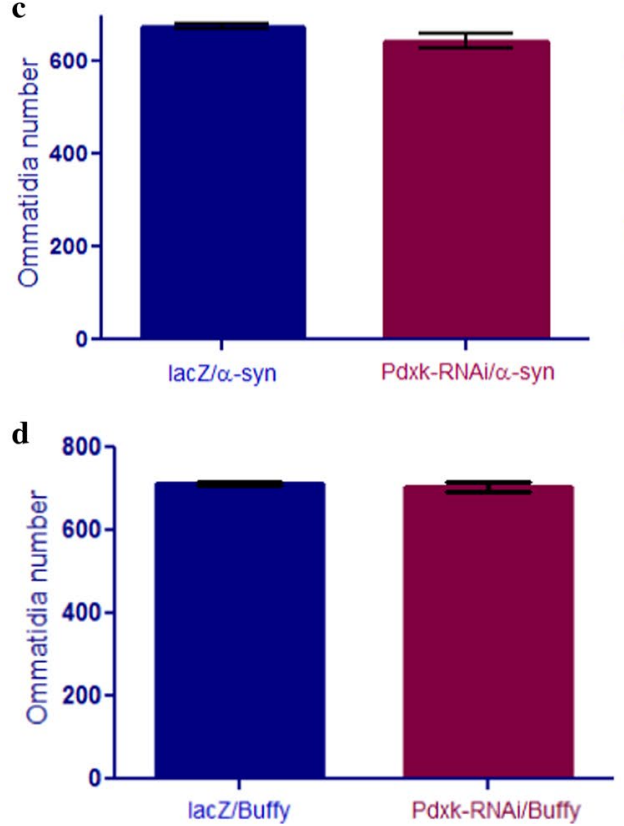

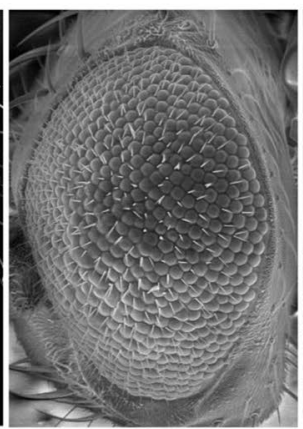

Pdxk-RNAi

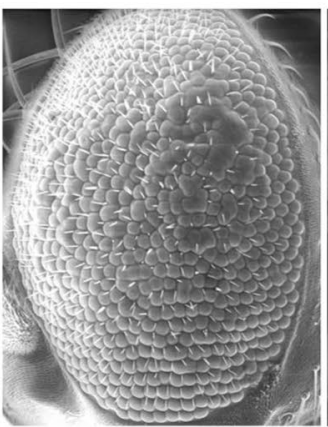

Pdxk-RNAi/a-syn

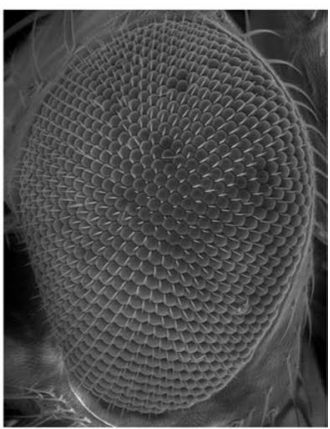

Pdxk-RNAi/Buffy
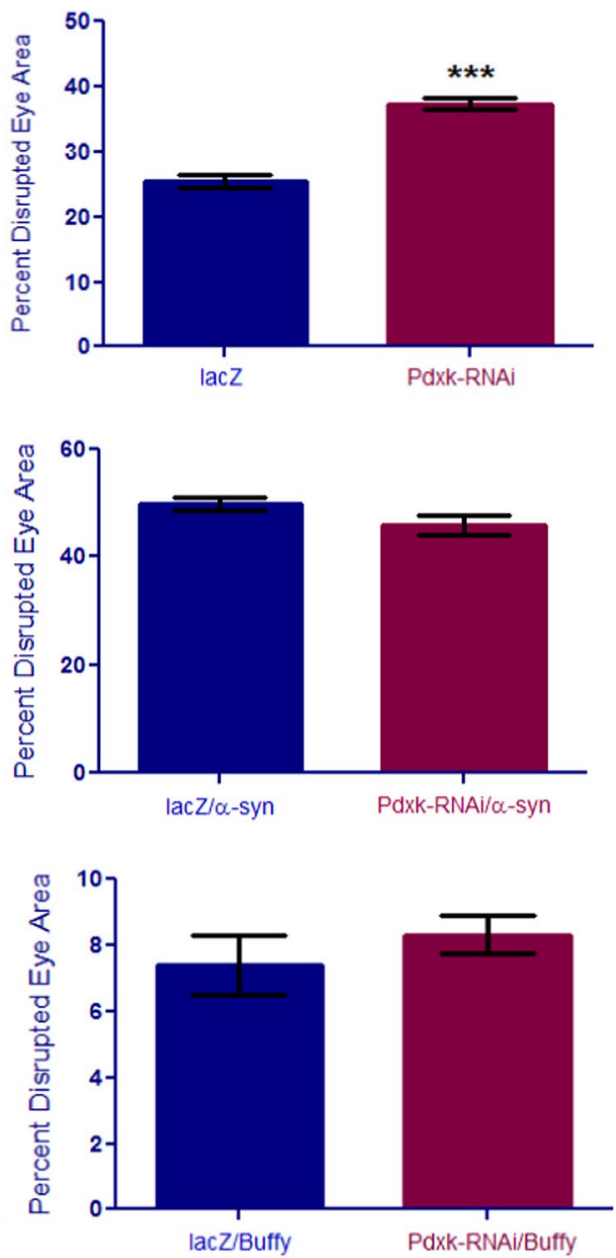

Fig. 5 The conditional expression of $P d x k$ in the Drosophila eye results in reduced ommatidia number and increased disruption of the eyes and is suppressed upon co-expression with Buffy. a Scanning electron micrographs when Pdxk is inhibited in the eye (I) GMR-Gal4/UAS-lacZ and (II) GMRGal4/Pdxk-RNAi. b The biometric analysis of the eyes indicated a slight decrease in mean ommatidia number and a higher percent disruption of the eye when compared to the control. c The co-expression of Pdxk-RNAi with a-synuclein-expression (I) UAS-a-synuclein; GMR-Gal4/UAS-IacZ and (II) UAS-a-synuclein; GMR-Gal4/Pdxk-RNAi. Biometric analysis of a-synuclein-expression and Pdxk inhibition in the developing eye revealed no significance in the number of ommatidia and the degree of ommatidial disruption. d Co-expression of Buffy with Pdxk-RNAi (I) UAS-Buffy; GMR-Gal4/UASlacZ and (II) UAS-Buffy; GMR-Gal4/Pdxk-RNAi. Biometric analysis showed restoration of the mean number of ommatidia and ommatidial disruption to control levels. Comparisons were determined by unpaired two-tailed T test $(P<0.05)$, error bars are SEM, $n=10$ and asterisks represent statistical significance 


\section{Discussion}

The directed inhibition of $P d x k$ in the $D d c$-Gal4-expressing neurons of $D$. melanogaster result in decreased lifespan and an age-dependent loss in climbing ability, phenotypes strongly associated with models of PD. Pyridoxal kinase is involved in the conversion of pyridoxal into pyridoxal-5'-phosphate (PLP), an important enzyme cofactor in intermediary metabolism [14]. These $\mathrm{B}_{6}$ vitamers seem to be involved in all vital cellular functions, from glucose metabolism to nucleic acid synthesis to being potent antioxidants. Our inhibition of this protein kinase in the Ddc-Gal4-expressing neurons resulted in shortened lifespan and impaired climbing ability, while in supportive experiments, its inhibition in the developing eye resulted in reduced ommatidia number and a high degree of ommatidial disarray. The involvement of PLP in the conversion of dopa to dopamine is an important step in its synthesis, it seems therefore, that decreased neuronal PLP can mimic the effects of decreased dopamine, a key step in PD development, and possibly of major impact in dopamine-containing cells. It is not surprising therefore, that decrease in the availability of such an important coenzyme by inhibition of the kinase function involved in its conversion results in defective neuronal function that may lead to neurodegeneration.

The expression of $\alpha$-synuclein in Ddc-Gal4-expressing neurons can result in PD-like characteristics in Drosophila [23]. We investigated the possible existence of a link between $\alpha$-synuclein-induced phenotypes and the inhibition of $P d x k$ in the $D d c$-Gal4-expressing neurons of Drosophila. Our results indicate that loss of $P d x k$ function in Ddc-Gal4-expressing neurons that were additionally expressing $\alpha$-synuclein did not enhance the $\alpha$-synuclein-induced phenotypes of decreased lifespan and impaired climbing ability. Similarly, when we conducted similar comparisons in the neuron rich Drosophila eye, we observed that inhibition of $P d x k$ did not alter the consequences of $\alpha$-synuclein expression in the developing eye. Therefore, the toxic effects of $\alpha$-synuclein are sufficient to generate phenotypes but inhibition of $P d x k$ activity does not confer any additional disadvantage. Alternatively, loss-of- $P d x k$-induced toxicity may precede the effects of $\alpha$-synuclein accumulation with the expression of $\alpha$-synuclein enhancing the $P d x k-R N A i$-induced phenotypes and specifically in the developing eye. This observation indicates a cell specific mechanism, where the expression of $\alpha$-synuclein in the Pdxk-RNAi background in the developing eye and not in the DA neurons enhances the phenotypes. Additionally, the multitude of biological functions that are dependent on PLP from a dysfunctional antioxidant activity to mitochondrial dysfunction may induce neurotoxicity in these sensitive neurons.
The directed expression of the pro-survival $B c l-2$ homologue Buffy with Pdxk RNA interference resulted in the suppression of the loss of $P d x k$-induced phenotypes of decreased survival and impaired locomotor ability. Buffy like many other pro-survival $\mathrm{Bcl}-2$ proteins, is thought to be a "guardian" of the mitochondria and confers survival advantages by restricting death-promoting molecules [38, 39]. Buffy suppressed the $P d x k$-induced phenotypes of reduced lifespan and locomotor ability and further restored ommatidia number and decreased the disruption of the ommatidial array. Not only do PLP deficiencies accelerate mitochondrial decay, but they increase ROS radicals $[15,16]$, a property that make neurons more vulnerable. It is possible that Buffy restores this balance by initiating or participation in pro-survival signals.

\section{Conclusions}

Although Pdxk has not been confirmed to be a PDcausative gene, it has been associated to increased risk of the disease. The inhibition of this gene activity by the directed expression of an RNAi transgene in the $D d c$ Gal4-expressing neurons phenocopies PD-like symptoms in Drosophila, and therefore may represent a novel model of PD. This appear to corroborate other studies that show several mechanisms involved in the aetiology of PD. More studies are required to chart out a pathway for $P d x k$ activity in Drosophila, and importantly to show at a molecular level the changes associated with the lossof-function of this kinase in the development and function of dopaminergic neurons.

\section{Methods}

\section{Bioinformatic analysis}

The protein sequences for Pyridoxal kinase were obtained from the National Center for Biotechnology Information (NCBI; http://www.ncbi.nlm.nih.gov/ protein/) and the domains were identified using the NCBI Conserved Domain Database (CDD; http://www. ncbi.nlm.nih.gov/cdd) [31] and the Eukaryotic Linear Motif resource (ELM; http://elm.eu.org/) [40] which focuses on annotation and detection of eukaryotic linear motifs (ELMs) or short linear motifs (SLiMs). The alignment of sequences was performed with Clustal Omega (http://www.ebi.ac.uk/Tools/msa/clustalo/) [33] to show conservation of the kinase domain in the queried organisms. The prediction of the nuclear export signal (NES) was by NetNES (http://www.cbs.dtu.dk/ services/NetNES/) [35]. The nuclear localisation signal (NLS) was predicted with cNLS Mapper [36]. The subcellular localisation was performed by MultiLoc2 [34] (https://abi.inf.uni-tuebingen.de/Services/MultiLoc2). Transmembrane domains were identified using TMpred 
[37], a program based on statistical analysis of TMbase (http://www.ch.embnet.org/software/TMPRED_form. html).

\section{Drosophila media and culture}

Stocks and crosses were maintained on a standard medium prepared from cornmeal, molasses, yeast, agar, water and treated with propionic acid and methylparaben to inhibit fungal growth. Stocks were maintained at $23 \pm 2{ }^{\circ} \mathrm{C}$ while crosses and experiments were carried out at 25 and $29^{\circ} \mathrm{C}$.

\section{Drosophila stocks and derivative lines}

UAS-Buffy [38] was generously supplied by Dr. Leonie Quinn (University of Melbourne), UAS- $\alpha$-synuclein [23] by Dr. Mel Feany of Harvard Medical School, and DdcGal4 [41] by Dr. Jay Hirsch (University of Virginia). The UAS-Pdxk-RNAi (P\{KK108240\}VIE-260B) [42] flies were obtained from Vienna Drosophila Resource Center while GMR-Gal4 [43] and UAS-lacZ flies were obtained from the Bloomington Drosophila Stock Center at Indiana University. The UAS- $\alpha$-synuclein/CyO; Ddc-Gal4/TM3; UAS- $\alpha$-synuclein/CyO; GMR-Gal4; UAS-Buffy/CyO; Ddc-Gal4; and UAS-Buffy/CyO; GMR-Gal4 complex lines were generated following a standard protocol as previously described $[44,45]$. They expressed $\alpha$-synuclein or overexpressed Buffy either in the Ddc-Gal4-expressing neurons using the Ddc-Gal4 transgene or in the developing eye using the GMR-Gal4 transgene. Analysis of complex lines was confirmed via PCR reactions and gel electrophoresis.

\section{Ageing assay}

Multiple matings of each genotype were carried out and male flies collected upon eclosion and analysed following a protocol that has been described [29, 46]. At least 200 flies were aged per genotype and scored every 2 days for the presence of deceased adults [47]. Longevity data was analysed using the GraphPad Prism version 5.04 and survival curves were compared using the Log-rank (Mantel-Cox) test with significance determined with a 95\% confidence interval, at a $\mathrm{P} \leq 0.05$ with Bonferroni correction.

\section{Climbing assay}

A batch of male flies was analysed for their ability to climb according to a standard protocol [48]. In brief, every 7 days after eclosion, 50 or fewer males of each genotype were assayed for their ability to climb. Climbing indices were computed and analysed with GraphPad Prism version 5.04. Climbing curves were fitted using non-linear regression and compared using 95\% confidence interval with a $0.05 \mathrm{P}$ value.

\section{Scanning electron microscopy of the Drosophila eye}

Male flies were prepared for scanning electron microscopy using a standard protocol as previously described [29]. Ten different eye images for each genotype were analysed using the National Institutes of Health (NIH) ImageJ software [49] and biometric analysis performed using GraphPad Prism version 5.04. The percentage disruption of the eye was calculated as previously described [50]. Statistical analysis comprised of unpaired student $\mathrm{T}$ test and one-way analyses of variance with NewmanKeuls multiple comparison post-test and significance determined at $\mathrm{P}$ values equal or less than 0.05 .

\section{Abbreviations \\ DA: dopaminergic; Ddc: dopa decarboxylase; GMR: glass multiple reporter; PD: Parkinson disease; Pdxk: pyridoxal kinase; PLP: pyridoxal-5'-phosphate; RNAi: ribonucleic acid interference; SEM: standard error of the mean. \\ Authors' contributions \\ PGM performed the bioinformatic, survival, climbing, biometric and statistical analyses. BES conceived and participated in the design, supervision of the study and revisions to the final draft of the manuscript. Both authors read and approved the final manuscript.}

\section{Acknowledgements}

Not applicable.

\section{Competing interests}

The authors declare that they have no competing interests.

\section{Availability of data and materials}

The datasets used and analyzed during the current study are available from the corresponding author on reasonable request.

\section{Ethics approval}

This study has been conducted under the approval of the Animal Care Committee of Memorial University of Newfoundland as a Category of Invasiveness Level A protocol under the project title of "Genetic, biochemical and molecular analysis of cell survival and cell death in Drosophila melanogaster" (Protocol Number: 15-09-BS). Consent was not required for this study.

\section{Funding}

PGM has been partially funded by Department of Biology Teaching Assistantships and a School of Graduate Studies Fellowship from Memorial University of Newfoundland. BES has been funded by a Natural Sciences and Engineering Research Council of Canada (NSERC) Discovery Grant. The funding bodies were not involved in the design of the study, collection, analysis, and interpretation of data nor in the writing the manuscript.

\section{Publisher's Note}

Springer Nature remains neutral with regard to jurisdictional claims in published maps and institutional affiliations.

Received: 24 February 2016 Accepted: 2 June 2017

Published online: 12 June 2017

\section{References}

1. Miller DB, O'Callaghan JP. Biomarkers of Parkinson's disease: present and future. Metabolism. 2015;64:6.

2. Dickson DW. Parkinson's disease and parkinsonism: neuropathology. Cold Spring Harb Perspect Med. 2012;2:a009258. 
3. Pfeiffer RF. Non-motor symptoms in Parkinson's disease. Parkinsonism Relat Disord. 2015. doi:10.1016/j.parkreldis.2015.09.004.

4. Jellinger KA. Neuropathology of sporadic Parkinson's disease: evaluation and changes of concepts. Mov Disord. 2012;27:8-30.

5. Forno LS. Neuropathology of Parkinson's disease. J Neuropathol Exp Neurol. 1996;55:259-72.

6. Bonifati V. Genetics of Parkinson's disease-state of the art, 2013. Parkinsonism Relat Disord. 2014;20(Suppl 1):8.

7. Houlden $\mathrm{H}$, Singleton AB. The genetics and neuropathology of Parkinson's disease. Acta Neuropathol. 2012;124:325-38.

8. Vanhauwaert R, Verstreken P. Flies with Parkinson's disease. Exp Neurol. 2015;274:42-51.

9. Yahr MD, Duvoisin RC. Pyridoxine and levodopa in the treatment of parkinsonism. JAMA. 1972;220:861

10. de Lau LML, Koudstaal PJ, Witteman JCM, Hofman A, Breteler MMB. Dietary folate, vitamin B12, and vitamin $\mathrm{B} 6$ and the risk of Parkinson disease. Neurology. 2006;67:315-8.

11. Murakami K, Miyake Y, Sasaki S, Tanaka K, Fukushima W, Kiyohara C, et al. Dietary intake of folate, vitamin B6, vitamin B12 and riboflavin and risk of Parkinson's disease: a case-control study in Japan. Br J Nutr. 2010;104:757-64.

12. Bowling FG. Pyridoxine supply in human development. Semin Cell Dev Biol. 2011;22:611-8.

13. Wu X-YY, Lu L. Vitamin B6 deficiency, genome instability and cancer. Asian Pac J Cancer Prev. 2012;13:5333-8.

14. Fitzpatrick TB, Amrhein N, Kappes B, Macheroux P, Tews I, Raschle T. Two independent routes of de novo vitamin B6 biosynthesis: not that different after all. Biochem J. 2007:407:1-13.

15. Ames BN, Atamna H, Killilea DW. Mineral and vitamin deficiencies can accelerate the mitochondrial decay of aging. Mol Aspects Med. 2005;26:363-78.

16. Depeint F, Bruce WR, Shangari N, Mehta R, O'Brien PJ. Mitochondrial function and toxicity: role of $B$ vitamins on the one-carbon transfer pathways. Chem Biol Interact. 2006;163:113-32.

17. Jain SK, Lim G. Pyridoxine and pyridoxamine inhibits superoxide radicals and prevents lipid peroxidation, protein glycosylation, and ( $\mathrm{Na}++\mathrm{K}+$ ATPase activity reduction in high glucose-treated human erythrocytes. Free Radic Biol Med. 2001;30:232-7.

18. Merrill AH, Henderson JM, Wang E, McDonald BW, Millikan WJ. Metabolism of vitamin B-6 by human liver. J Nutr. 1984;114:1664-74.

19. McCormick DB, Chen $\mathrm{H}$. Update on interconversions of vitamin B-6 with its coenzyme. J Nutr. 1999;129:325-7.

20. Elstner M, Morris CM, Heim K, Lichtner P, Bender A, Mehta D, et al. Single-cell expression profiling of dopaminergic neurons combined with association analysis identifies pyridoxal kinase as Parkinson's disease gene. Ann Neurol. 2009;66:792-8.

21. Guella I, Asselta R, Tesei S, Zini M, Pezzoli G, Duga S. The PDXK rs2010795 variant is not associated with Parkinson disease in Italy. Ann Neurol. 2010;67:411-2 (author reply 412).

22. Staveley BE. Drosophila models of Parkinson disease. In: LeDoux MS, editor. Movement disorders: genetics and models. 2nd ed. New York: Elsevier Science; 2015. p. 345-54.

23. Feany MB, Bender WW. A Drosophila model of Parkinson's disease. Nature. 2000;404:394-8

24. Buttner S, Broeskamp F, Sommer C, Markaki M, Habernig L, AlavianGhavanini A, et al. Spermidine protects against alpha-synuclein neurotoxicity. Cell Cycle. 2014;13:3903-8.

25. Kong Y, Liang X, Liu L, Zhang D, Wan C, Gan Z, et al. High throughput sequencing identifies microRNAs mediating alpha-synuclein toxicity by targeting neuroactive-ligand receptor interaction pathway in early stage of Drosophila Parkinson's disease model. PLoS ONE. 2015;10:e0137432.

26. Zhu ZJ, Wu KC, Yung WH, Qian ZM, Ke Y. Differential interaction between iron and mutant alpha-synuclein causes distinctive Parkinsonian phenotypes in Drosophila. Biochim Biophys Acta. 2016;1862:518-25.

27. Botella JAA, Bayersdorfer F, Gmeiner F, Schneuwly S. Modelling Parkinson's disease in Drosophila. Neuromolecular Med. 2009:11:268-80.
28. Brand AH, Perrimon N. Targeted gene expression as a means of altering cell fates and generating dominant phenotypes. Development. 1993:118:401-15.

29. M'Angale PG, Staveley BE. The BCl-2 homologue Buffy rescues alphasynuclein-induced Parkinson disease-like phenotypes in Drosophila. BMC Neurosci. 2016;17:24.

30. M'Angale PG, Staveley BE. The HtrA2 Drosophila model of Parkinson disease is suppressed by the pro-survival BCl-2 Buffy. Genome. 2017;60:1-7.

31. Marchler-Bauer A, Derbyshire MK, Gonzales NR, Lu S, Chitsaz F, Geer LY, et al. CDD: NCBl's conserved domain database. Nucleic Acids Res. 2015:43:D222-6.

32. Dinkel H, Van Roey K, Michael S, Davey NE, Weatheritt RJ, Born D, et al. The eukaryotic linear motif resource ELM: 10 years and counting. Nucleic Acids Res. 2013. doi:10.1093/nar/gkt1047.

33. Sievers F, Wilm A, Dineen D, Gibson TJ, Karplus K, Li W, et al. Fast, scalable generation of high-quality protein multiple sequence alignments using clustal omega. Mol Syst Biol. 2011;7:539.

34. Blum T, Briesemeister S, Kohlbacher O. MultiLoc2: integrating phylogeny and gene ontology terms improves subcellular protein localization prediction. BMC Bioinform. 2009:10:274.

35. la Cour T, Kiemer L, Mølgaard A, Gupta R, Skriver K, Brunak S. Analysis and prediction of leucine-rich nuclear export signals. Protein Eng Des Sel. 2004;17:527-36.

36. Kosugi S, Hasebe M, Tomita M, Yanagawa H. Systematic identification of cell cycle-dependent yeast nucleocytoplasmic shuttling proteins by prediction of composite motifs. Proc Natl Acad Sci USA. 2009;106:10171-6.

37. Artimo P, Jonnalagedda M, Arnold K, Baratin D, Csardi G, de Castro E, et al. ExPASy: SIB bioinformatics resource portal. Nucleic Acids Res. 2012:40:W597-603.

38. Quinn L, Coombe M, Mills K, Daish T, Colussi P, Kumar S, et al. Buffy, a Drosophila Bcl-2 protein, has anti-apoptotic and cell cycle inhibitory functions. EMBO J. 2003;22:3568-79.

39. Igaki T, Miura M. Role of Bcl-2 family members in invertebrates. Biochim Biophys Acta. 2004;1644:73-81.

40. Dinkel H, Van Roey K, Michael S, Kumar M, Uyar B, Altenberg B, et al. ELM 2016 - data update and new functionality of the eukaryotic linear motif resource. Nucleic Acids Res. 2016:44:D294-300.

41. Li H, Chaney S, Roberts IJ, Forte M, Hirsh J. Ectopic G-protein expression in dopamine and serotonin neurons blocks cocaine sensitization in Drosophila melanogaster. Curr Biol. 2000;10:211-4.

42. Dietzl G, Chen D, Schnorrer F, Su K-CC, Barinova Y, Fellner M, et al. A genome-wide transgenic RNAi library for conditional gene inactivation in Drosophila. Nature. 2007;448:151-6.

43. Freeman M. Reiterative use of the EGF receptor triggers differentiation of all cell types in the Drosophila eye. Cell. 1996;87:651-60.

44. M'Angale PG, Staveley BE. BCl-2 homologue Debcl enhances alpha-synuclein-induced phenotypes in Drosophila. PeerJ. 2016;4:e2461.

45. M'Angale PG, Staveley BE. Inhibition of Atg6 and Pi3K59F autophagy genes in neurons decreases lifespan and locomotor ability in Drosophila melanogaster. Genet Mol Res. 2016;15:gmr15048953.

46. Todd AM, Staveley BE. Expression of Pink1 with alpha-synuclein in the dopaminergic neurons of Drosophila leads to increases in both lifespan and healthspan. Genet Mol Res. 2012;11:1497-502.

47. Staveley BE, Phillips JP, Hilliker AJ. Phenotypic consequences of copperzinc superoxide dismutase overexpression in Drosophila melanogaster. Genome. 1990;33:867-72.

48. Todd AM, Staveley BE. Novel assay and analysis for measuring climbing ability in Drosophila. Drosoph Inf Serv. 2004;87:101-7.

49. Schneider CA, Rasband WS, Eliceiri KW. NIH Image to ImageJ: 25 years of image analysis. Nat Methods. 2012;9:671-5.

50. M'Angale PG, Staveley BE. Effects of a-synuclein expression in the developing Drosophila eye. Drosoph Inf Serv. 2012;95:85-9. 